\title{
Experimental Analysis on the Accelerating Rheological Failure Process of Soft Soil Based on the Catastrophe Theory
}

\author{
Hua $\mathrm{HU}^{1,2, a}$, Liang $\mathrm{CAl}^{1, \mathrm{~b}^{*}}$, Jian $\mathrm{CHENG}^{2, \mathrm{c}}$, Xiang-Hua $\mathrm{LI}^{2, \mathrm{~d}}$ \\ ${ }^{1}$ College of architecture and civil engineering of Xiamen University, Fujian Xiamen 361005 \\ ${ }^{2}$ Xiamen University Tan Kah Kee College, Fujian Zhangzhou City, 363105 \\ axmhuhua@xujc.com,,cailiang1109@163.com,,287932217@qq.com,,844929099@qq.com
}

${ }^{*}$ Corresponding author

Keywords: Catastrophe Theory, Dynamic Load, Soft Rock-soil, Accelerating Rheology, Failure Instability.

\begin{abstract}
The rheologic action of the soft rock-soil could be accelerated by the dynamic load, which induces catastrophe instability failure and more geo-technical engineering accidents and geologic disasters. Marine deposit soft-soil in Xiamen region is taken as the test sample, of which the rheological dynamics characteristics are tested under the dynamic load by adopting the dynamic rheological tri-axial device; the failure process of the accelerating rheological catastrophe is analyzed based on the catastrophe theory. The research shows that such different changing stages as decelerating rheology, relatively stable rheology and accelerating rheology are manifested under the process of the different amplitude dynamic load, and the catastrophe way in the accelerating rheological process is the gradual failure instability. The research results is of important and theoretical significance in revealing the dynamic rheological characteristics of the soft rock-soil, exploring the accelerating rheological failure process and conditions of catastrophe instability; they are also of important and practical significance in improving and ameliorating the indicator-monitoring system and technical method for the geo-technical geological disaster, enhancing the forecast scientific and accuracy.
\end{abstract}

\section{Introduction}

There are 2 kinds of variations in the objective world: One is smooth, continuous, and incessant; the other is discontinuous leap such as rocks rupture, bridges collapse, slope instability, ear thquake, etc. In order to study the discontinuous change process including "catastrophe", mathematical theories like topology, singularity, stability, etc. have been applied to the research, and catastrophe theory come into being. Catastrophe theory has become most advanced research direction in the geotechnical engineering.

Soft rock-soil has rheological properties, the rheological behavior is too slow to bring serious deformation under the static loads, but easy to accelerate rheologic action into a geotechnical instability and more serious geo-technical engineering accidents and geologic disasters such as landslide, collapse, land subsidence, foundation slide instability, engineering inclined crack, etc. under the external dynamic loads such as earthquake, mechanical vibration, vehicular traffic, engineering blasting, etc[1,2]. Therefore, the study of soft rock-soil's accelerating rheological failure process and conditions of catastrophe instability is of important and theoretical significance in exploring the rheological dynamics mechanism and rheological instability dynamics condition; It's also of important and practical significance in improving and ameliorating the indicator-monitoring system and technical method for the geo-technical geological disaster, enhancing the forecast scientific and accuracy.

\section{The Cusp Catastrophe Model Theory}

Catastrophe theory mainly inspects a system or process from one stable state leaps to another stable state. The state one system is in can be described by a set of parameters: some certain 
parameters will acquire a only extremum if system is stable, and not stable if there are more than one extremum in a certain range. Therefore, in order to assess the system's stability, extremum of function must be calculated which means what make the derivative of the function equal to zero must be calculated as singularity, or the critical point $[3,4]$.

Most discontinuous phenomena in the nature and social phenomenon can be expressed by some specific geometry. The simplest catastrophic model is Cusp Catastrophe when there are 2 controlled variables. It's easy to establish the critical surface of Cusp Catastrophe which is geometrically readable and widely used in rock-soil mechanics. [5,6]

\section{The Dynamic Rheological Test of Marine Deposit Soft-soil}

Specimens are made from marine deposit soft-soil in HaiCang, Xiamen by pressure method and tested under the sinusoidal dynamic load by adopting the motor controlled dynamic tri-axial device from Company GDC in England. Specimens will be saturated and loaded into pressure chamber, then consolidated under $100 \mathrm{kPa}$ confining pressure until consolidation is stable, finally ready for rheological test under sinusoidal dynamic loads step by step with a condition of drainage. The relationship between rheological strain (different load frequency and amplitude) and time will be studied. The next load can be applied to the test sample when the strain tends to be stable or there is no obvious rheological failure symptom until it occurs rheological failure. In the test, failure symptom can be confirmed when strain occurs a big increment or accumulated strain is over $5 \%$. Load frequency is $0.01 \mathrm{~Hz}, 0.1 \mathrm{~Hz}$, and amplitude is $10,20,40,60 \mathrm{kPa}$.

Tab. 1 Testing Program of Frequency and Max-amplitude of Dynamic Loading of Sinusoidal Variation

\begin{tabular}{|c|c|c|c|c|c|}
\hline $\begin{array}{l}\text { Test } \\
\text { number }\end{array}$ & $\begin{array}{c}\text { Dynamic } \\
\text { Frequency } \\
/[\mathrm{Hz}]\end{array}$ & $\begin{array}{c}\text { maximum } \\
\text { dynamic } \\
\text { loading / } \\
\sigma_{\mathrm{d}}[\mathrm{kPa}]\end{array}$ & $\begin{array}{c}\text { Test } \\
\text { number }\end{array}$ & $\begin{array}{c}\text { Dynamic frequency } \\
/[\mathrm{Hz}]\end{array}$ & $\begin{array}{c}\underset{\text { dynamic }}{\operatorname{maximum}} \\
\text { loading } / \sigma_{\mathrm{d}}[\mathrm{kPa}]\end{array}$ \\
\hline 1 & \multirow{4}{*}{0.01} & 10 & 5 & \multirow{4}{*}{0.1} & 10 \\
\hline 2 & & 20 & 6 & & 20 \\
\hline 3 & & 40 & 7 & & 40 \\
\hline 4 & & 60 & 8 & & 60 \\
\hline
\end{tabular}

Fig. 1 shows the relationship curve between axes dynamic strain and time under different dynamic load frequency and illustrates that: in the whole test, samples show a tendency of change from relatively stable rheology to accelerating rheology. It's relatively stable rheology where strain grows slowly under a low stress range $(10 \sim 20 \mathrm{kPa})$, while accelerating rheology where rheological displacement gradually increases till the failure when load amplitude is over $40 \mathrm{kPa}$.
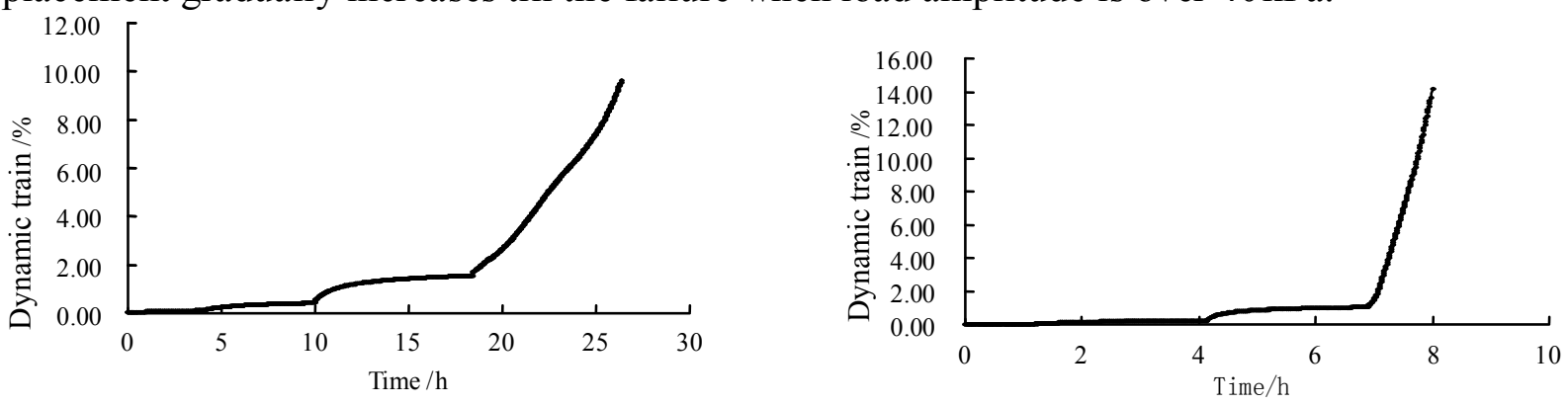

Fig. 1 Relationship Curve between Axes Dynamic Strain and Time under Different Dynamic Loading Frequency

When $\mathrm{f}=0.01 \mathrm{~Hz}$, maximum dynamic strain is $0.12 \%$ when dynamic load amplitude is $10 \mathrm{kPa}$ and 150 times; maximum dynamic strain is $0.43 \%$ when dynamic load amplitude is $20 \mathrm{kPa}$ and 210 times; maximum dynamic strain is $1.56 \%$ when dynamic load amplitude is $40 \mathrm{kPa}$ and 300 times 
where accelerating rheology started; maximum dynamic strain is $9.62 \%$ when dynamic load amplitude is $60 \mathrm{kPa}$ and 300 times where accelerating rheology was conspicuous till the failure.

When $\mathrm{f}=0.1 \mathrm{~Hz}$, maximum dynamic strain is $0.03 \%$ when dynamic load amplitude is $10 \mathrm{kPa}$ and 500 times; maximum dynamic strain is $0.21 \%$ when dynamic load amplitude is $20 \mathrm{kPa}$ and 600 times; maximum dynamic strain is $1.04 \%$ when dynamic load amplitude is $40 \mathrm{kPa}$ and 650 times where accelerating rheology started; maximum dynamic strain is $14.14 \%$ when dynamic load amplitude is $60 \mathrm{kPa}$ and 1200 times where accelerating rheology was conspicuous till the failure.

\section{Process Analysis of Accelerating Rheology Cusp Catastrophe of Soft Rock-soil}

Stress-strain backbone curve. According to the failure criterion (maximum strain reach 5\%), the stress-strain backbone curve of sample 1 has been chosen and cut by $7.3405 \%$ of strain. The fitting expression is:

$$
\sigma=0.4353 \varepsilon^{3}-6.7665 \varepsilon^{2}+32.877 \varepsilon+8.8748
$$

Stress-strain backbone curve of sample 2 has also been chosen and cut by $5.16 \%$ of strain. The fitting expression is:

$$
\sigma=0.7601 \varepsilon^{3}-8.0887 \varepsilon^{2}+27.645 \varepsilon+7.3689
$$

Analysis of cusp catastrophe failure based on tri-axial rheology test.

Sample 1: $\sigma=0.4353 \varepsilon^{3}-6.7665 \varepsilon^{2}+32.877 \varepsilon+8.8748$

1st derivative calculation: $\sigma^{\prime}=1.3059 \varepsilon^{2}-13.533 \varepsilon+32.877$

2nd derivative calculation: $\sigma^{\prime \prime}=2.6118 \varepsilon-13.533$; Let: $\sigma^{\prime \prime}=0$, then $\varepsilon_{1}=5.1815$;

Do Taylor series expansion to three at $\varepsilon_{1}$ by $\sigma=0.4353 \varepsilon^{3}-6.7665 \varepsilon^{2}+32.877 \varepsilon+8.8748$;

Then

$$
\sigma=0.4353(\varepsilon-5.1815)^{3}-2.1835(\varepsilon-5.1815)+58.1161
$$

Substitute $\varepsilon_{1}=5.1815$ and equation (3) to potential energy function of Unit volume:

$$
\begin{aligned}
& V=\int_{0}^{t} \sigma d \varepsilon \\
& V=0.1088\left(\varepsilon-\varepsilon_{1}\right)^{4}-1.09175\left(\varepsilon-\varepsilon_{1}\right)^{2}+58.1161\left(\varepsilon-\varepsilon_{1}\right)+252.0154
\end{aligned}
$$

Let $x=\left(\varepsilon-\varepsilon_{1}\right) / \varepsilon_{1}$; and ignore the influence of constant term, then

$$
V=78.4243\left(x^{4}-0.3738 x^{2}+3.8397 x\right)
$$

Let $V_{1}(x)=x^{4}-0.3738 x^{2}+3.8397 x$ and $V_{1}(x)^{\prime}=0$, then

Equilibrium function of Cusp Catastrophe model: $4 x^{3}-0.7476 x+3.8397=0$

Derivative calculation at each side, gives singular set: $12 x^{2}-0.7476=0$

The solution is $x= \pm 0.2496$, corresponding strain is $\varepsilon_{a}=3.880, \varepsilon_{b}=6.4748$. 


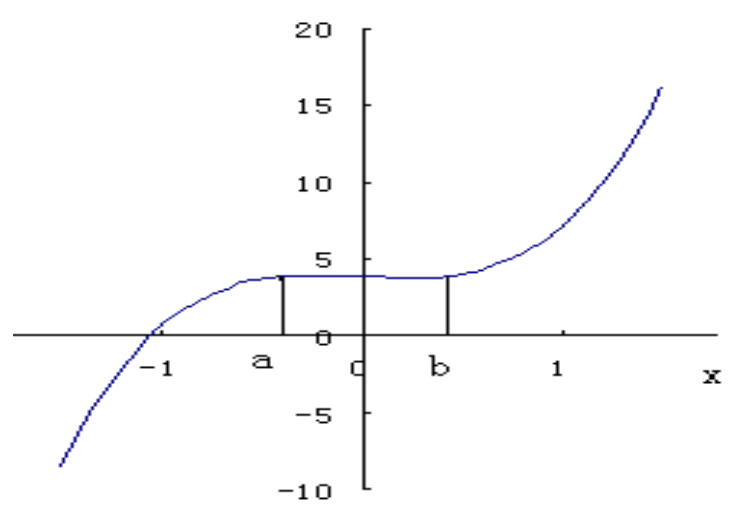

Fig. 2 Controlling Curve of Sample 1

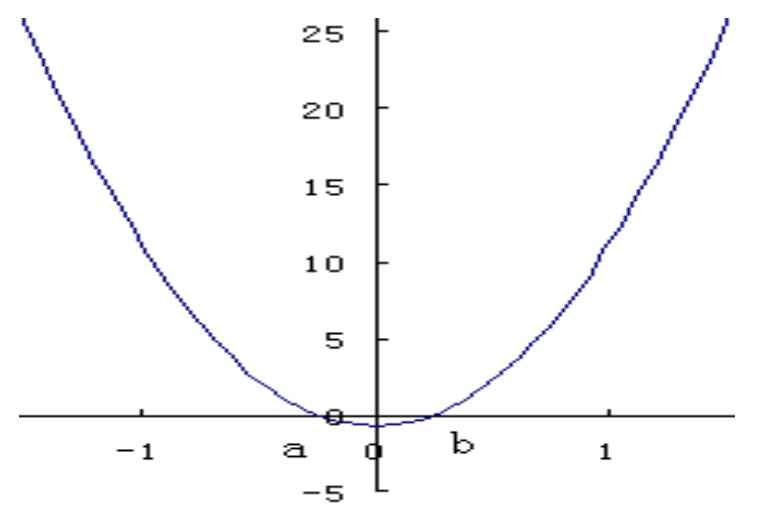

Fig. 3 Equilibrium Curve of Sample 1

According to fig. $2 \&$ fig. 3 , a, b is singular point and at the 4th stage of the test's result instead of middle of the surface, that is to say, the mode failure is gradual without catastrophe since it dissatisfied the equilibrium function of Cusp Catastrophe model.

Sample 2: $\sigma=0.7601 \varepsilon^{3}-8.0887 \varepsilon^{2}+27.645 \varepsilon+7.3689$

Do the same calculation, then Equilibrium function of Cusp Catastrophe model: $4 \mathrm{x}^{3}-0.438 \mathrm{x}+4.431=0$; singular set: $12 x^{2}-0.438=0$

The solution is $x= \pm 0.1910$, corresponding strain is $\varepsilon_{a}=2.870, \varepsilon_{b}=4.225$.

According to fig. 4 \& fig.5, a, b is singular point and at the 4 th stage of the test's result instead of middle of the surface, that is to say, the mode failure is gradual without catastrophe since it dissatisfied the equilibrium function of Cusp Catastrophe model.

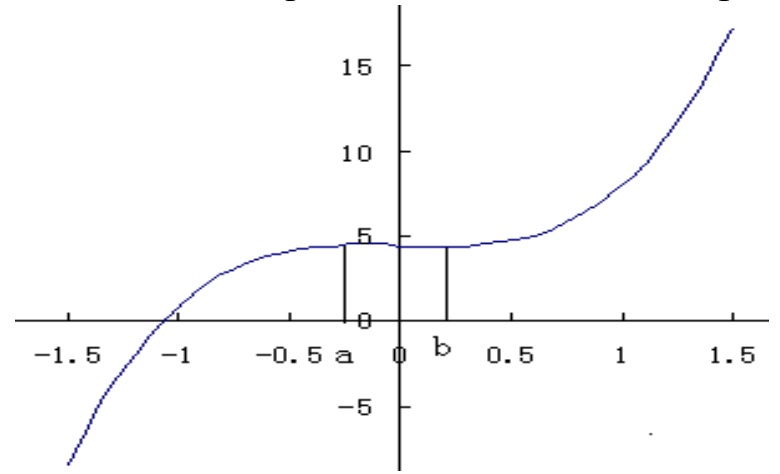

Fig.4 Controlling Curve of Sample 2

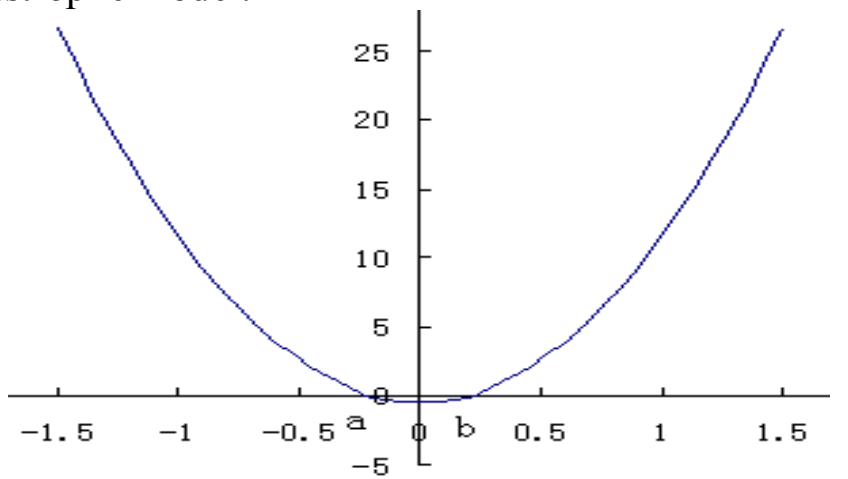

Fig.5 Equilibrium Curve of Sample 2

\section{Summary}

This paper, based on Cusp Catastrophe theory, explores and analyses the failure process of the dynamic rheological and failure mechanism. The simplest catastrophic model is Cusp Catastrophe when there are 2 controlled variables. It's easy to establish he critical surface of Cusp Catastrophe which is geometrically readable. Therefore, Cusp Catastrophe model is selected to analysis for process of rheological failure. After calculation of equilibrium function of Cusp Catastrophe model and singular set, we conclude that singular point is at the 4th stage of the test's result instead of middle of the surface, that is to say, the mode failure is gradual without catastrophe since it dissatisfied the equilibrium function of Cusp Catastrophe model which fitted the test.

\section{Acknowledgement}

This research was financially supported by the National Natural Science Foundation of China (51278437) and Natural Science Foundation of Department of Science \& Technology of Zhang Zhou city in Fu Jian Provincial (ZZ2013J17). 


\section{Reference}

[1]HU Hua. The rheological model and rheological equation of sullage soft soil under dynamic loading[J]. Rock and Soil Mechanics, 2007, 28(2).

[2]HU Hua,GU Heng-xing,YU Deng-rong. Research on dynamic rheological characteristics and rheologic parameters of sullage soft soil [J]. Rock and Soil Mechanics, 2008, 29(3).

[3]Zhang Xiao-tao, Dou Lin-ming, Li Zhi-hua. Model for coal-rock body's creep and mutation [J]. China Coal, 2005, 31(1).

[4]Zuo Yu-jun, Li Xi-bing. Catastrophic model and testing study on failure of static loading rock system under dynamic loading [J]. Chinese Journal of Rock Mechanics and Engineering, 2005, 24(5).

[5]Liu jun, Qin Si-qing. Study on catastrophic model with cusp point for failure of stratified rock mass with a gentle inclination [J]. Chinese Journal of Geotechnical Engineering, 2001, 23(1).

[6]Long hui, Qin Si-qing . Nonlinear dynamic model and catastrophe analysis of slope evolution [J]. Journal of Engineering Geology, 2001, 9(3). 